\section{(1) \\ CrossMark}

\title{
Design of pulmonary rehabilitation programmes during acute exacerbations of COPD: a systematic review and network meta-analysis
}

\author{
Ana Machado $0^{1,2}$, Pedro Matos Silva ${ }^{3}$, Vera Afreixo ${ }^{2,4}$, Cátia Caneiras $\mathbb{1}^{5,6}$, \\ Chris Burtin ${ }^{7,8}$ and Alda Marques ${ }^{1,2}$
}

@ERSpublications

Combination of exercise training, breathing techniques and education and psychosocial support is the most effective design to deliver pulmonary rehabilitation during acute exacerbations of COPD https:// bit.ly/2TAh2aP

Cite this article as: Machado A, Matos Silva $\mathrm{P}$, Afreixo V, et al. Design of pulmonary rehabilitation programmes during acute exacerbations of COPD: a systematic review and network meta-analysis. Eur Respir Rev 2020; 29: 200039 [https://doi.org/10.1183/16000617.0039-2020].

ABSTRACT This systematic review aimed to systematise the different designs used to deliver pulmonary rehabilitation during acute exacerbations of COPD (AECOPD) and explore which ones are the most effective. PubMed, Scopus, Web of Science, EBSCO and Cochrane were searched. Randomised controlled trials comparing pulmonary rehabilitation or at least one of its components with usual care or comparing different components of pulmonary rehabilitation were included. Network meta-analysis was conducted in MetaXL 5.3 using a generalised pairwise modelling framework. Pooled effects compared each treatment to usual care.

42 studies were included. Most studies were conducted in an inpatient setting (57\%) and started the intervention 24-48 h after hospital admission (24\%). Exercise training (71\%), education and psychosocial support (57\%) and breathing techniques (55\%) were the most used components. Studies combining exercise with breathing techniques presented the larger effects on exercise capacity (weighted mean difference (WMD) -41.06, 95\% CI -131.70-49.58) and health-related quality of life (WMD 14.64, 95\% CI 8.73-20.54), and breathing techniques presented the larger effects on dyspnoea (WMD 1.90, 95\% CI 0.53-3.27) and length of hospitalisation (effect size $=0.15,95 \%$ CI $-0.28-0.57$ ). A few minor adverse events were found.

Pulmonary rehabilitation is a safe intervention during AECOPD. Exercise, breathing techniques, and education and psychosocial support seem to be the core components for implementing pulmonary rehabilitation during AECOPD. Studies may now focus on comparisons of optimal timings to start the intervention, total duration of the intervention, duration and frequency of sessions, and intensity for exercise prescription.

\section{Introduction}

COPD is a progressive disease that is often punctuated by exacerbations, defined as episodes of acute worsening of respiratory symptoms that result in additional therapy $[1,2]$. Acute exacerbations of COPD

This article has been revised according to the author correction published in the March 2021 issue and the erratum published in the June 2021 issue of the European Respiratory Review.

This article has supplementary material available from err.ersjournals.com

Submitted article, peer reviewed

Part of this work was submitted as an abstract to the 2018 ERS International Congress.

Received: 07 Feb 2020 | Accepted after revision: 14 May 2020

Copyright $\odot$ ERS 2020. This article is open access and distributed under the terms of the Creative Commons Attribution Non-Commercial Licence 4.0. 
(AECOPD) impact negatively on patients' health status and disease progression, and increase patients' susceptibility to more exacerbations, hospitalisations and death $[1,2]$. Treatment goals for patients with AECOPD are, therefore, to minimise the negative impact of these events and prevent their recurrence [1].

Pulmonary rehabilitation is a comprehensive intervention presenting well-established benefits in patients with stable COPD $[1,3]$. It has been shown to: 1) improve exercise capacity, muscle strength, functional capacity and health-related quality of life (HRQoL); 2) reduce symptoms, hospitalisations and unscheduled healthcare visits; and 3) enhance self-management and self-efficacy [3]. Given these benefits, it would seem reasonable to consider pulmonary rehabilitation as a management strategy for AECOPD [4]. However, studies assessing the role of pulmonary rehabilitation during AECOPD have shown inconsistent results $[1,2,5,6]$. One of the reasons for this inconsistency is the heterogeneity in the design of pulmonary rehabilitation programmes. Studies have been delivering pulmonary rehabilitation programmes in diverse settings (i.e. inpatient, outpatient, community, home), with different components implemented in isolation or in combination (e.g. aerobic training, muscle strengthening, education) and with a wide variety of durations, intensities and timings (i.e. the time chosen to integrate patients into the pulmonary rehabilitation programme) [5]. It is known that all these aspects may influence efficacy of pulmonary rehabilitation [5]. Thus, this systematic review aimed to systematise the different designs used to deliver pulmonary rehabilitation during AECOPD and explore which ones are the most effective.

\section{Methods}

Search strategy

Searches in the Cochrane Library and the International Prospective Register of Systematic Reviews (PROSPERO) were conducted prior to the development of the present systematic review to exclude the existence of reviews or protocols with the same purpose as the present one. Since no similar studies were found, the systematic review protocol was registered at PROSPERO (www.crd.york.ac.uk/prospero registration no. CRD42017056930).

A systematic literature search was performed in February 2017 on the following electronic databases: PubMed, Scopus, Web of Science, EBSCO and Cochrane. Additionally, weekly automatic updates retrieved from the databases were performed until March 2020. Search terms were based on a combination of the following keywords: ("acute exacerbation of chronic obstructive pulmonary disease" OR "AECOPD" OR "acute exacerbation of COPD" OR "exacerbation of COPD") AND ("pulmonary rehabilitation" OR "respiratory rehabilitation" OR "physiotherapy” OR "physical therapy” OR "respiratory physiotherapy” OR "respiratory physical therapy" OR "exercise" OR "exercise training” OR "breathing exercise" OR "breathing technique" OR "airway clearance" OR "walking” OR "aerobic training" OR "resistance training" OR "balance training" OR "flexibility training" OR "stretch training" OR "strength training" OR "education" OR "psychoeducation" OR "psychosocial support"). The search was limited to terms found in titles, abstracts and keywords. Additionally, the reference lists of the selected articles and key systematic reviews were scanned for other potential eligible studies.

\section{Eligibility criteria and study selection}

Studies were included if they: 1) studied patients within 3 weeks of AECOPD onset [1, 4, 7] or until 2 weeks after discharge [8]; 2) used pulmonary rehabilitation or at least one of its components (i.e. any kind of exercise training, breathing techniques, airway clearance techniques or education and psychosocial support) as an intervention; 3) were written in English, French, Portuguese or Spanish; and 4) were randomised controlled trials. Studies were admissible independently of the treatment setting or degree of severity of the AECOPD. Randomised controlled trials comparing pulmonary rehabilitation or at least one of its components with usual care or comparing different components of pulmonary rehabilitation were considered. Abstracts in conference proceedings were excluded. After removing duplicates, two reviewers assessed all the potential studies identified. Studies were selected based on their titles and abstracts; when

Affiliations: ${ }^{1}$ Respiratory Research and Rehabilitation Laboratory (Lab 3R), School of Health Sciences (ESSUA), University of Aveiro, Aveiro, Portugal. ${ }^{2}$ Institute for Biomedicine (iBiMED), University of Aveiro, Aveiro, Portugal. ${ }^{3}$ Unidade Local de Saúde de Matosinhos, Matosinhos, Portugal. ${ }^{4}$ Center for Research and Development in Mathematics and Applications, Dept of Mathematics, University of Aveiro, Aveiro, Portugal. ${ }^{5}$ Healthcare Dept, Nippon Gases Portugal, Vila Franca de Xira, Portugal. ${ }^{6}$ Environmental Health Institute (ISAMB), Faculty of Medicine, Universidade de Lisboa, Lisbon, Portugal. ${ }^{7}$ REVAL - Rehabilitation Research Center, Faculty of Rehabilitation Sciences, Hasselt University, Diepenbeek, Belgium. ${ }^{8}$ BIOMED - Biomedical Research Institute, Hasselt University, Diepenbeek, Belgium.

Correspondence: Alda Marques, Respiratory Research and Rehabilitation Laboratory, School of Health Sciences (ESSUA), University of Aveiro, Agras do Crasto - Campus Universitário de Santiago, Edifício 30 , 3810-193, Aveiro, Portugal. E-mail: amarquesवua.pt 
the title and abstract were relevant to the purpose of the review, the full-text article was read carefully to decide on its inclusion. A third reviewer was consulted to solve any disagreements. This systematic review was reported according to the Preferred Reporting Items for Systematic reviews and Meta-analyses (PRISMA) guidelines [9].

\section{Quality assessment and data extraction}

Two reviewers independently assessed the quality of the included studies with the Delphi List, a list composed of nine items [10]. The total score consists of summing the number of items that are satisfied (i.e. evaluated as yes) and ranges from 0 to 9 [11]. Studies with a total score $\geqslant 5$ were considered to be of high quality $[11,12]$.

Data from the included studies were extracted to a structured table format comprising the following topics: study (first author, year of publication, country); population (number of participants, age, sex, forced expiratory volume in $1 \mathrm{~s}\left(\mathrm{FEV}_{1}\right)$ ); timing and setting; type of intervention; duration of the intervention and intensity of each of its components; duration and frequency of sessions; outcomes and outcome measures; and results. All noninvasive outcome measures (e.g. no blood analysis or biopsies) were considered. Corresponding authors of the included studies were contacted via e-mail to request additional information about the interventions and/or data (e.g. means and standard deviations) when required. All data were retrieved from the published articles, supplementary files and authors' contacts.

\section{Data analysis and synthesis}

Inter-rater agreement analysis using Cohen's kappa was used to explore the consistency of the quality assessment performed by the two reviewers. The value of Cohen's kappa ranges from 0 to 1 and can be interpreted as slight $(\leqslant 0.2)$, fair $(0.21-0.4)$, moderate $(0.41-0.6)$, substantial $(0.61-0.8)$, or almost perfect $(\geqslant 0.81)$ agreement [13]. The statistical analysis was performed using IBM SPSS 24.0.

Studies were grouped accordingly to the component (i.e. exercise training, education and psychosocial support, breathing techniques) or a combination of components used in their intervention. Only studies that included at least exercise training and education and psychosocial support [4] were considered to have performed pulmonary rehabilitation. Due to the large diversity of outcome measures and units of measurement used, and the wide variety of interventions implemented, it was only possible to conduct network meta-analysis with a limited number of variables, and comparisons were only possible between components and duration of the interventions. Intervention programmes were considered to be either short-term programmes, when the total duration of their intervention was $<8$ weeks, or long-term programmes, if the intervention lasted $\geqslant 8$ weeks [4]. Only studies performing more than one session in their interventions were considered in the network meta-analysis. For comparisons between components of the intervention, studies that performed at least exercise (i.e. exercise training or pulmonary rehabilitation) were considered in the same subgroup. For variables that did not fit in the network meta-analysis, effect sizes (ES) were computed whenever possible. Individual ES were calculated as Cohens' $\mathrm{d}$ based on the pre/post-means and standard deviations, according to the formula of MoRRIS [14], and were interpreted as small $(\geqslant 0.2)$, medium $(\geqslant 0.5)$ or large $(\geqslant 0.8)$ [15]. Network meta-analysis was conducted using MetaXL 5.3, which implements the generalised pairwise modelling framework for meta-analysis [16]. This method involves: 1) pooling effect sizes for direct comparisons between each combination of two interventions using meta-analysis; 2) performing indirect comparisons by automated generation of all possible closed loops of three treatments of which one is common to two studies; and 3) pooling direct and indirect effects using meta-analysis to give a final effect size comparing each treatment to the common comparator, i.e. control (standard of care) [16]. Pooled effect estimates were calculated assuming a random-effects model. Input data for the 6-min walk test (6MWT), the modified Borg scale and the St George's respiratory questionnaire (SGRQ) were the standardised mean difference in each group and the respective standard deviation, either directly retrieved from the study or estimated [17]. Output data were the weighted mean difference (WMD) and corresponding confidence intervals. Regarding the length of hospitalisation, input data were the mean and standard deviation of each study, and output data were the pooled Cohen's d value and corresponding confidence intervals. Statistical heterogeneity across pooled direct effects was assessed using Cochran's Q and the H index. Transitivity was assessed by examining inconsistency across the network using the weighted pooled $\mathrm{H}$ index, with values $<3$ being considered to indicate minimal inconsistency [16].

\section{Results}

Study selection and quality assessment

The literature search provided a total of 6029 records. After removal of duplicates, 5484 records were screened for relevant content using titles and abstracts. From these, 5396 were excluded. The full-text of 88 potentially relevant articles was assessed and 64 articles were excluded due to the following reasons: 1) studies 
were not randomised controlled trials $(n=24) ; 2)$ articles were written in German $(n=3)$ or Italian $(n=1)$; 3 ) were abstracts only $(n=9)$; 4) studies did not include pulmonary rehabilitation or any of its components as an intervention $(n=8) ; 5)$ studies did not include participants with AECOPD $(n=3)$ or presented the results for this population independently $(n=2)$; and 6) studies did not include participants within 3 weeks of the AECOPD onset or until 2 weeks after discharge $(n=14)$. An additional 18 studies were retrieved from the search for relevant articles within the reference lists $(n=10)$ and the weekly automatic updates $(n=8)$. Thus, a total of 42 studies were included in this review and 25 were used for meta-analysis (figure 1).

Results of the methodological quality assessment are presented in table 1 . Most of the studies $(n=33$; $78.6 \%)$ were high quality. The agreement between the two reviewers was substantial ( $\mathrm{k}=0.80$ (95\% CI $0.64-$ $0.97), \mathrm{p}<0.001$; percentage of agreement $85.3 \%)$.
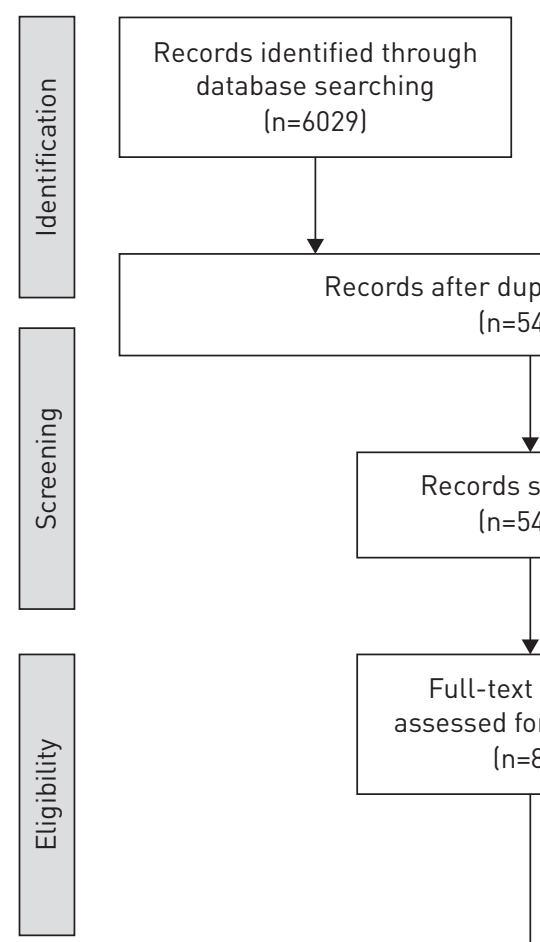

Additional records identified through other sources

$$
(n=18)
$$
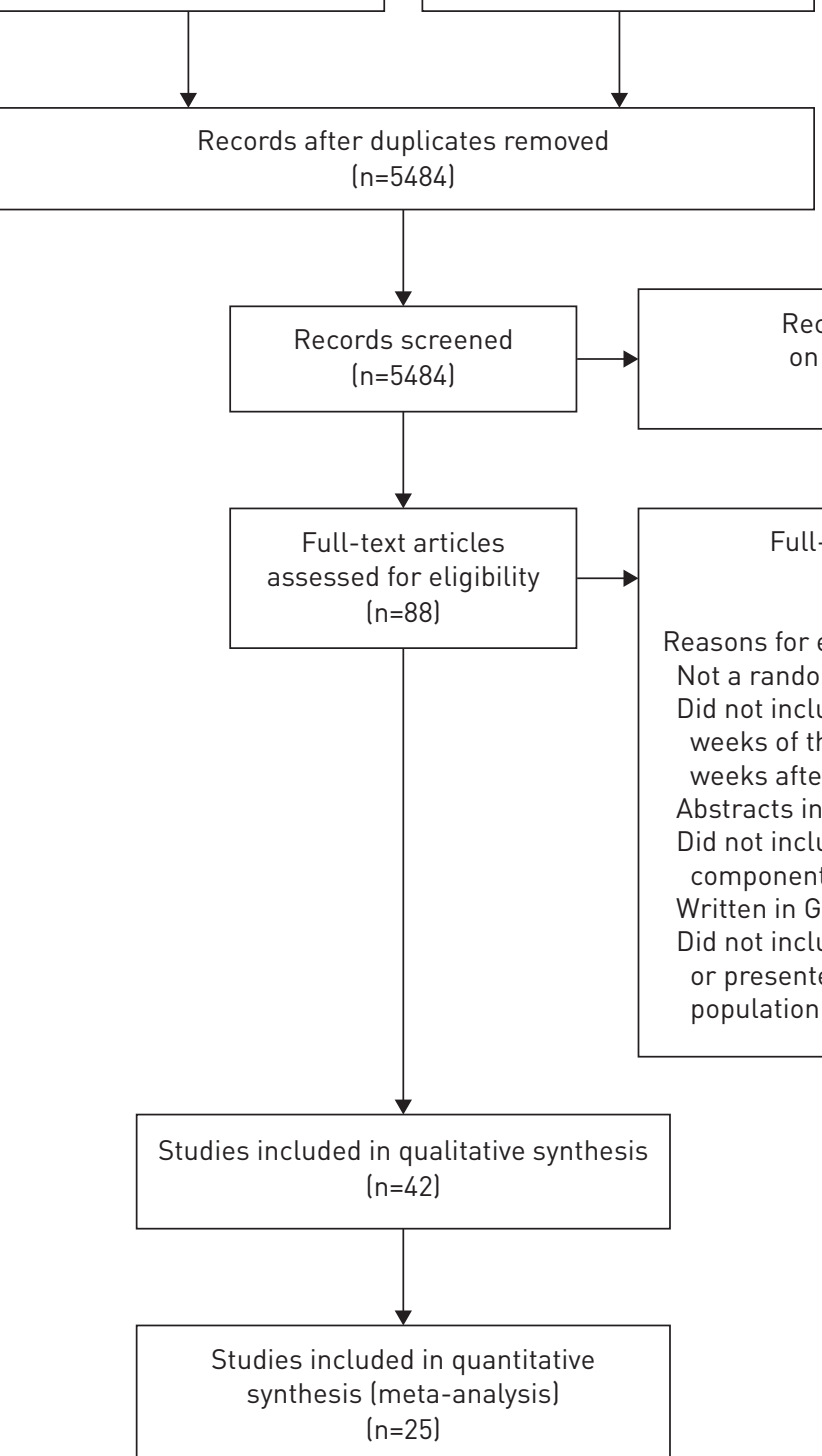

FIGURE 1 PRISMA flowchart of the included studies. AECOPD: acute exacerbation of COPD; PR: pulmonary rehabilitation. 


\begin{tabular}{|c|c|c|c|c|c|c|c|c|c|c|}
\hline First author [ref.] & Randomisation & $\begin{array}{l}\text { Concealed } \\
\text { allocation }\end{array}$ & $\begin{array}{l}\text { Groups } \\
\text { similar }\end{array}$ & $\begin{array}{l}\text { Eligibility } \\
\text { criteria }\end{array}$ & $\begin{array}{l}\text { Assessor } \\
\text { blinded }\end{array}$ & $\begin{array}{l}\text { Care provider } \\
\text { blinded }\end{array}$ & $\begin{array}{l}\text { Patient } \\
\text { blinded }\end{array}$ & $\begin{array}{l}\text { Point estimates and } \\
\text { measures of variability }\end{array}$ & $\begin{array}{l}\text { Intention-to-treat } \\
\text { analysis }\end{array}$ & Total \\
\hline KIRSTEN [32] & Yes & Don't know & Yes & Yes & Don't know & No & No & Yes & No & $4 / 9$ \\
\hline NAVA [37] & Yes & Don't know & Yes & Yes & Don't know & Don't know & Don't know & Yes & No & $4 / 9$ \\
\hline BELLONE [54] & Yes & No & Yes & Yes & Don't know & Don't know & Don't know & Yes & No & $4 / 9$ \\
\hline HeRnandez [56] & Yes & No & Yes & Yes & Don't know & Don't know & Yes & Yes & No & $5 / 9$ \\
\hline Yohannes [52] & Yes & Don't know & Yes & Yes & Yes & Yes & Yes & Yes & No & $7 / 9$ \\
\hline MAN [35] & Yes & Don't know & Yes & Yes & No & No & No & Yes & Yes & $5 / 9$ \\
\hline MURPHY [36] & Yes & Yes & Yes & Yes & Don't know & Don't know & No & Yes & No & $5 / 9$ \\
\hline CASAS [19] & Yes & No & No & Yes & Don't know & Don't know & Yes & Yes & Yes & $5 / 9$ \\
\hline GARCIA-AYMERICH [47] & Yes & No & Yes & Yes & Don't know & No & Yes & Yes & No & $5 / 9$ \\
\hline EATON [29] & Yes & Yes & Yes & Yes & Yes & No & No & Yes & Yes & $7 / 9$ \\
\hline KodRIC [48] & Yes & Don't know & Don't know & Yes & Don't know & Don't know & Don't know & Yes & No & $3 / 9$ \\
\hline$B_{A B U}[25]$ & Yes & Don't know & No & Yes & No & No & Yes & Yes & No & $4 / 9$ \\
\hline Seymour [38] & Yes & Yes & Yes & Yes & No & Don't know & No & Yes & Yes & $6 / 9$ \\
\hline TROOSTERS [43] & Yes & Yes & Yes & Yes & No & No & No & Yes & No & $5 / 9$ \\
\hline Ko [33] & Yes & Don't know & Yes & Yes & Yes & No & No & Yes & Yes & $6 / 9$ \\
\hline CRoss 2012 [20] & Yes & Yes & Yes & Yes & Don't know & Don't know & No & Yes & Yes & $6 / 9$ \\
\hline Puhan [23] & Yes & Yes & Yes & Yes & Don't know & Don't know & Don't know & Yes & Yes & $6 / 9$ \\
\hline TANG [40] & Yes & Don't know & Yes & Yes & Yes & No & Don't know & Yes & Yes & $6 / 9$ \\
\hline Chaplin [27] & Yes & Don't know & Ye & Yes & No & No & Yes & Yes & No & $5 / 9$ \\
\hline LAINSCAK [57] & Yes & Yes & Yes & Yes & No & No & No & Yes & No & $6 / 9$ \\
\hline ALI [24] & Yes & Don't know & Yes & Yes & Don't know & No & No & Yes & No & $4 / 9$ \\
\hline BoRgES [26] & Yes & Yes & Yes & Yes & Yes & No & No & Yes & No & $6 / 9$ \\
\hline Deepak [28] & Yes & No & Yes & Yes & Don't know & Don't know & Don't know & Yes & No & 4/9 \\
\hline GREULICH [30] & Yes & Yes & Yes & Yes & Yes & Yes & Don't know & Yes & No & $7 / 9$ \\
\hline OsAdNIK [22] & Yes & Yes & Yes & Yes & Yes & No & No & Yes & No & $6 / 9$ \\
\hline Song [39] & Yes & Don't know & Yes & Yes & No & No & Yes & Yes & No & $5 / 9$ \\
\hline VALenza [55] & Yes & Don't know & Yes & Yes & Don't know & No & Don't know & Yes & Yes & $5 / 9$ \\
\hline $\mathrm{HE}[31]$ & Yes & Don't know & No & Yes & Don't know & Don't know & Don't know & Yes & No & $3 / 9$ \\
\hline LIAO [34] & Yes & Don't know & Yes & Yes & Yes & No & No & Yes & No & $5 / 9$ \\
\hline Benzo [18] & Yes & No & Yes & Yes & Yes & No & Don't know & Yes & Yes & $6 / 9$ \\
\hline EASTWOOD [49] & Yes & Don't know & Yes & Yes & Yes & No & No & Yes & No & $5 / 9$ \\
\hline $\begin{array}{l}\text { JOHNSON-W WRRINGTON } \\
\text { [21] }\end{array}$ & Yes & Yes & Yes & Yes & Yes & No & No & Yes & Yes & $7 / 9$ \\
\hline TORRES-SÁNCHEZ [42] & Yes & Don't know & Yes & Yes & Yes & No & No & Yes & No & $5 / 9$ \\
\hline BASRI [50] & Yes & No & No & Yes & Yes & No & Yes & No & No & 4/9 \\
\hline TORRES -SÁNCHEZ [41] & Yes & Yes & Yes & Yes & Yes & No & Don't know & Yes & No & $6 / 9$ \\
\hline AвоumataR [58] & Yes & No & Yes & Yes & Yes & No & No & Yes & Yes & $6 / 9$ \\
\hline JanAUDIS-FERREIRA [59] & Yes & Don't know & Yes & Yes & Yes & No & No & Yes & No & $5 / 9$ \\
\hline Lopez-Lopez [53] & Yes & Yes & Yes & Yes & Don't know & No & No & Yes & No & $5 / 9$ \\
\hline TORRES-SÁNCHEz [51] & Yes & Yes & Yes & Yes & Don't know & No & No & Yes & No & $5 / 9$ \\
\hline LOPEZ-LOPEZ [46] & Yes & Yes & Yes & Yes & Yes & No & No & Yes & Yes & $7 / 9$ \\
\hline LOPEZ-LOPEZ [45] & Yes & Yes & Yes & Yes & Yes & No & No & Yes & No & $6 / 9$ \\
\hline KJJERGAARD [44] & Yes & Yes & Yes & Yes & No & No & No & Yes & Yes & $6 / 9$ \\
\hline
\end{tabular}




\section{Study characteristics}

Characteristics of the included studies are shown in table S1 and Appendix 1. The 42 studies included were conducted in 19 different countries. Almost $50 \%$ of the studies were published in the past 5 years. Six (14.2\%) of the included studies were multicentre [18-23].

In total, 3569 patients with a mean age of 69.1 years and a mean $\mathrm{FEV}_{1}$ of $39.4 \%$ predicted (data gathered from 35 studies, as seven of the included studies did not report the mean $\mathrm{FEV}_{1} \%$ pred) were enrolled in the included studies.

Studies evaluated the effect of the applied intervention on patients' exercise capacity ( $\mathrm{n}=23$, ES range $-1.8-$ 5.0) $[21,22,24-44]$, muscle strength $(\mathrm{n}=11$, ES -0.5-1.5) [26, 27, 36-38, 40-43, 45, 46], lung function $(\mathrm{n}=17, \mathrm{ES}-3.0-1.4)[22,24-26,30,32,33,37,39,40,42,46-51]$, functionality (n=7, ES -1.7-6.8) [31, 40, $41,45,46,52,53]$, physical activity levels ( $\mathrm{n}=3$, ES 1.2-2.6) [26, 41, 47], vital signs and peripheral oxygen saturation ( $\mathrm{n}=10, \mathrm{ES}-3.1-3.4)[24,29,31,32,36,37,39,42,48,50]$, symptoms ( $\mathrm{n}=26, \mathrm{ES}-4.0-4.8)$ [20-25, $28,29,31-34,36,37,42,45-55]$, impact of disease $(\mathrm{n}=4$, ES $-5.1-0.9)[30,31,44,49]$, disease severity $(\mathrm{n}=3$, ES -4.7--0.3) [22, 29, 31], HRQoL ( $\mathrm{n}=25$, ES -3.0-6.1) [18, 20-24, 26, 28-31, 33, 35, 36, 38, 39, 42, $46-48,51,55-58]$, length of stay ( $\mathrm{n}=19$, ES -0.8-0.9) [20, 22, 25, 26, 30, 36, 37, 40-43, 45, 46, 48, 49, 51-53, 56], new exacerbations ( $\mathrm{n}=4$, ES 0.1-1.0) [23, 33, 36, 38], hospital readmissions ( $\mathrm{n}=14$, ES 0.1-1.8) [19, 21, $22,29,33,35,38,43,44,47,52,56-58]$, and mortality (n=10, ES 0.1-1.2) [18, 19, 21, 22, 37, 44, 54, 56-58]. Additionally, studies also assessed patients' knowledge ( $\mathrm{n}=4$, ES $-0.3-15.1)[21,47,56,59]$, satisfaction $(\mathrm{n}=4$, ES 0.2-9.3) [47, 49, 56, 59], and self-efficacy ( $n=1$, ES -0.2) [21]. Nine studies assessed patients' adherence to the intervention $[18,26,29,33,35,38-40,44]$ and 16 studies explored the adverse effects associated with the intervention [20-22, 26, 27, 29-31, 35, 40, 41, 43, 45, 48, 49, 58].

\section{Programme designs}

A huge heterogeneity was found among the design of the intervention delivered in the different studies.

Most studies were conducted in an inpatient setting $(\mathrm{n}=24)[20,22,25-27,30-32,34,37,40-43,45,46$, 48-55], followed by inpatients plus patients' home $(n=6)[18,19,47,57-59]$, hospital outpatient departments $(n=3)[28,33,38]$, patients' home $(n=3)[21,36,56]$, inpatients plus hospital outpatient departments $(n=3)[23,24,29]$, community setting $(n=1)$ [35], and inpatients plus hospital outpatient departments plus patients' home $(n=1)$ [39]. In one study [44], patients could either be treated at a hospital outpatient department, a community setting or at home, depending on their physical status.

Studies started the intervention at a wide variety of timings, ranging from the time of admission up to 2-3 weeks after discharge. Most frequent timings to begin intervention were $24-48 \mathrm{~h}$ after hospital admission $(n=10)[22,30,31,40,41,45,46,49,53,57]$, after hospital discharge $(n=5)[28,33,35,38,56]$, at the moment of hospital discharge $(n=4)[18,19,36,47]$ and at hospital admission $(n=3)[25,43,55]$.

The total duration of intervention ranged from one session to 12 weeks. Frequency of sessions ranged from two sessions per week to five sessions per day. Duration of sessions varied from $<15$ min to $2 \mathrm{~h}$.

With regard to intervention components, 14 studies [19, 25, 27, 30, 32, 41, 45, 47, 48, 52, 54, 55, 58, 59] used a single component in the intervention whilst the other $28[18,20-24,26,28,29,31,33-40,42-44$, $46,49-51,53,56,57]$ applied a combination of components. Education and psychosocial support $(\mathrm{n}=24)$ $[18-24,26,28,29,31,34,35,38,39,44,46,47,49,50,56-59]$ was the most used component, followed by aerobic training $(\mathrm{n}=23)[18,21-24,27-29,31-41,44,45,52,53]$, strength training $(\mathrm{n}=23)[18,21,23,24$, $26,28-31,33-38,40,42-44,46,51,53,56]$ and breathing techniques(i.e. breathing control and/or airway clearance techniques) $(\mathrm{n}=23)[18,20,22,25,26,28,30,31,34,37,39,40,42,43,48-51,53-57] .71 \%$ $(n=30)$ of the studies used exercise training as part of the intervention. Other additional components used were electrostimulation $(n=4)[27,45,46,53]$, positive expiratory pressure therapy performed with noninvasive ventilation $[22,54]$ or devices $(n=3)[49]$, home diaries $(n=2)[35,36]$, respiratory muscle training $(\mathrm{n}=1)$ [37], nutrition $(\mathrm{n}=1)$ [28] and whole body vibration $(\mathrm{n}=1)$ [30]. Only $16(38.1 \%)$ of the included studies $[18,21-24,26,28,29,31,34,35,38,39,44,46,56]$ used pulmonary rehabilitation as an intervention if the pulmonary rehabilitation definition is taken into account [4].

17 studies [22, 24, 26, 27, 30-33, 36, 37, 40, 41, 43-45, 49, 53] presented information about the intensity of the intervention and, from these, seven [24, 26, 31, 37, 40,41,43] also described the training progression. Exercise training was performed based on patients' level of dyspnoea and fatigue, with modified Borg scale scores ranging from 3 to 7 [26, 31, 36, 37, 41, 44]. Aerobic training was prescribed at $40-85 \%$ of the maximal or submaximal test result $[24,31-33,37,40,44]$ and strength training at $40-80 \%$ of 1-repetition maximum [24, 26, 40,43,44]. Additional components used were: electrostimulation, which was applied using a range of frequencies from $12-50 \mathrm{~Hz}[27,30,45,53]$, with $50 \mathrm{~Hz}$ being the most used 
frequency; positive expiratory pressure therapy which was performed using $10-20 \mathrm{cmH}_{2} \mathrm{O}$ [22, 49]; and respiratory muscle training which was set at $50 \%$ of maximal inspiratory pressure [37].

Exercise training progressions were based on patients' symptoms at Borg or modified Borg scale $(\mathrm{n}=5)$ [26, $31,40,41,43$ ] and presenting clinical condition $(n=1)$ [37]. Increments in the aerobic training intensity were performed by increasing the total time of aerobic exercise $(n=3)[31,40,41]$, decreasing rest periods $(n=1)$ [24] and increasing the velocity and workload $(n=1)$ [41]. Strength training intensity was incremented by increasing the resistance used $(n=3)[24,26,40]$ or the number of sets $(n=1)$ [31].

Studies defined adherence to intervention as attendance to $50-75 \%$ of sessions, which was accomplished by $40-95 \%$ of the enrolled patients.

\section{Intervention effects}

The effects of the applied interventions on the different outcomes are summarised in table S1 and Appendix 1.

Exercise capacity was analysed using the 6MWT. Five comparisons against control were performed (figure 2a). The combination of exercise with breathing techniques was the intervention presenting the largest WMD (-41.06, 95\% CI -131.70-49.58), but no significant differences were found (figure 2b). The combination of education and psychosocial support with breathing techniques was inferior to control (WMD 108.98, 95\% CI 54.69-163.26) (figure 2b). Regarding the combination of exercise with breathing techniques, short-term programmes were superior to control (WMD -63.36, 95\% CI -87.67--39.06) and presented a larger WMD than long-term programmes (WMD -38.68, 95\% CI -92.76-15.41), although the latter was not statistically significant (figure 2c).

The modified Borg scale was used to analyse dyspnoea and four comparisons against control were performed (figure 3a). Breathing techniques was the intervention that performed better (WMD 1.90, 95\% CI 0.53-3.27) (figure 3b). The use of education and psychosocial support alone performed worse than control (WMD $-2.78,95 \% \mathrm{CI}-4.38--1.18$ ) (figure $3 \mathrm{~b}$ ).

HRQoL was analysed with the SGRQ total score. Five comparisons against control were performed (figure 4a). The combination of exercise with breathing techniques (WMD 14.64, 95\% CI 8.73-20.54) and exercise alone (WMD 12.62, 95\% CI 7.16-18.08) were the interventions presenting the larger WMD and were both superior to control (figure $4 \mathrm{~b}$ ).

Regarding to length of hospitalisation, five comparisons against control were performed (figure 5a). Breathing techniques (ES 0.15, 95\% CI -0.28-0.57) and the combination of exercise with breathing techniques (ES $0.11,95 \%$ CI $-0.28-0.50$ ) were the interventions presenting the larger effects, but no significant differences were found among interventions (figure $5 b$ ).

From the 16 studies exploring the adverse effects of their interventions, only four (29\%) [20, 22, 26, 40] found any adverse events, which were mainly increased dyspnoea or oxygen desaturation. Only three patients suffered more serious adverse events (i.e. dizziness and palpitations [22], vocal cord dysfunction [22] and chest pain [40]), which were justified by underlying comorbidities.

\section{Discussion}

This systematic review provided a comprehensive overview on the designs of pulmonary rehabilitation programmes implemented during AECOPD and was the first study to investigate which of those designs are the most effective to implement during this period of the disease.

Most programmes were conducted in hospitalised patients, were started at different timings of the AECOPD, integrated components commonly used in stable patients with COPD and were implemented during different lengths with a wide variety of frequency, duration, intensity and progressions of sessions.

Studies started their interventions at a variety of different time points in the patient pathway, making it difficult to establish the best time to start pulmonary rehabilitation. Early rehabilitation programmes, i.e. programmes that were started during hospital admission or at hospital discharge, were the most commonly used, probably because symptoms of AECOPD usually last for just 7 to 10 days [1].

The most used components in the interventions were aerobic training, strength training, breathing techniques and education and psychosocial support, either in isolation $[19,25,27,30,32,41,45,47,48$, $52,54,55,58,59]$ or in combination [18, 20-24, 26, 28, 29, 31, 33-40, 42-44, 46, 49-51, 53, 56, 57], both of which are also common in pulmonary rehabilitation practice with patients with stable COPD [60]. Nevertheless, during AECOPD, patients present an increased airway and systemic inflammation that results in increased dyspnoea, cough and sputum volume and purulence, and further impairment of muscle strength, functionality and exercise tolerance and, hence, different characteristics and needs from 

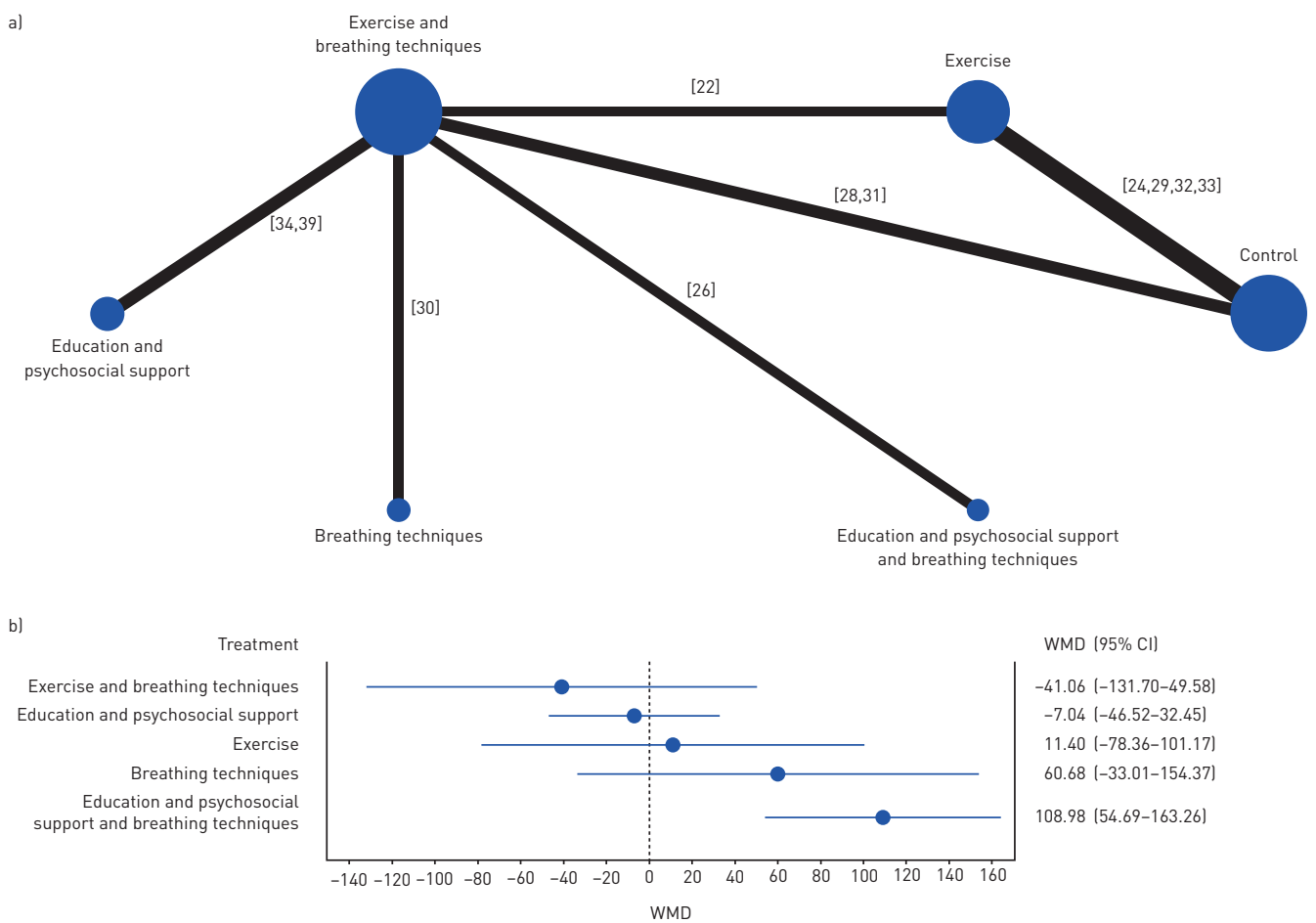

c)

WMD $(95 \% \mathrm{Cl}) \quad$ Weight $\%$

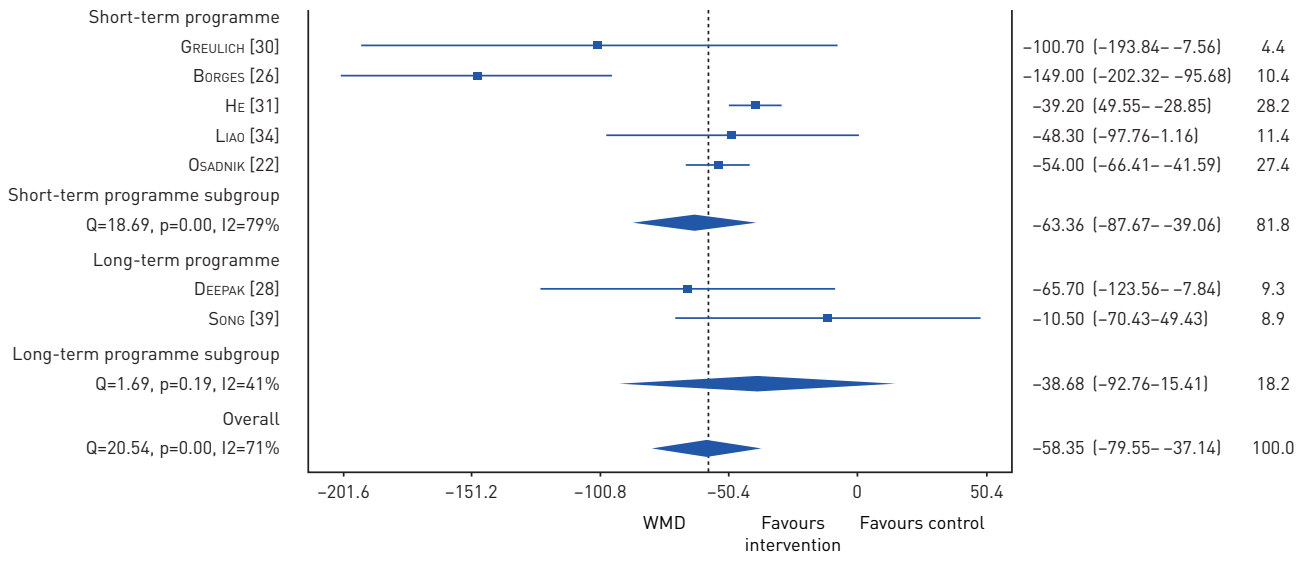

FIGURE 2 Effects of the different interventions on exercise capacity measured with 6-min walk test. a) Network plot of treatments with nodes representing the number of interventions being compared and edges representing the available direct comparisons (comparisons evaluated in at least one study) between pairs of interventions. b) Forest plot of network meta-analysis results (all interventions versus standard of care). c) Forest plot of comparison of short-term programmes versus long-term programmes using a combination of exercise with breathing techniques. WMD: weighted mean difference.

those in the stable phase $[1,61,62]$. It is, therefore, likely that the core components of pulmonary rehabilitation programmes during AECOPD will be different from those established for stable patients. Overall pooled results showed that combining exercise with breathing techniques is the most effective intervention to improve exercise capacity during AECOPD and the only design that results in improvements above the minimal clinically important difference of $30 \mathrm{~m}$ established for the 6MWT [63], although this has been established for patients who are stable and not for patients with AECOPD. Additionally, the combination of exercise with breathing techniques, followed by exercise, were the most effective interventions to improve HRQoL, being superior to standard of care and resulting in improvements above the minimal clinically important difference of 4 points (also established for stable patients) in the SGRQ [64]. Breathing techniques were shown to be the most effective intervention to reduce dyspnoea in patients with AECOPD, being superior to standard of care and resulting in improvements above the minimal clinically important difference of 0.9 points established for the modified 

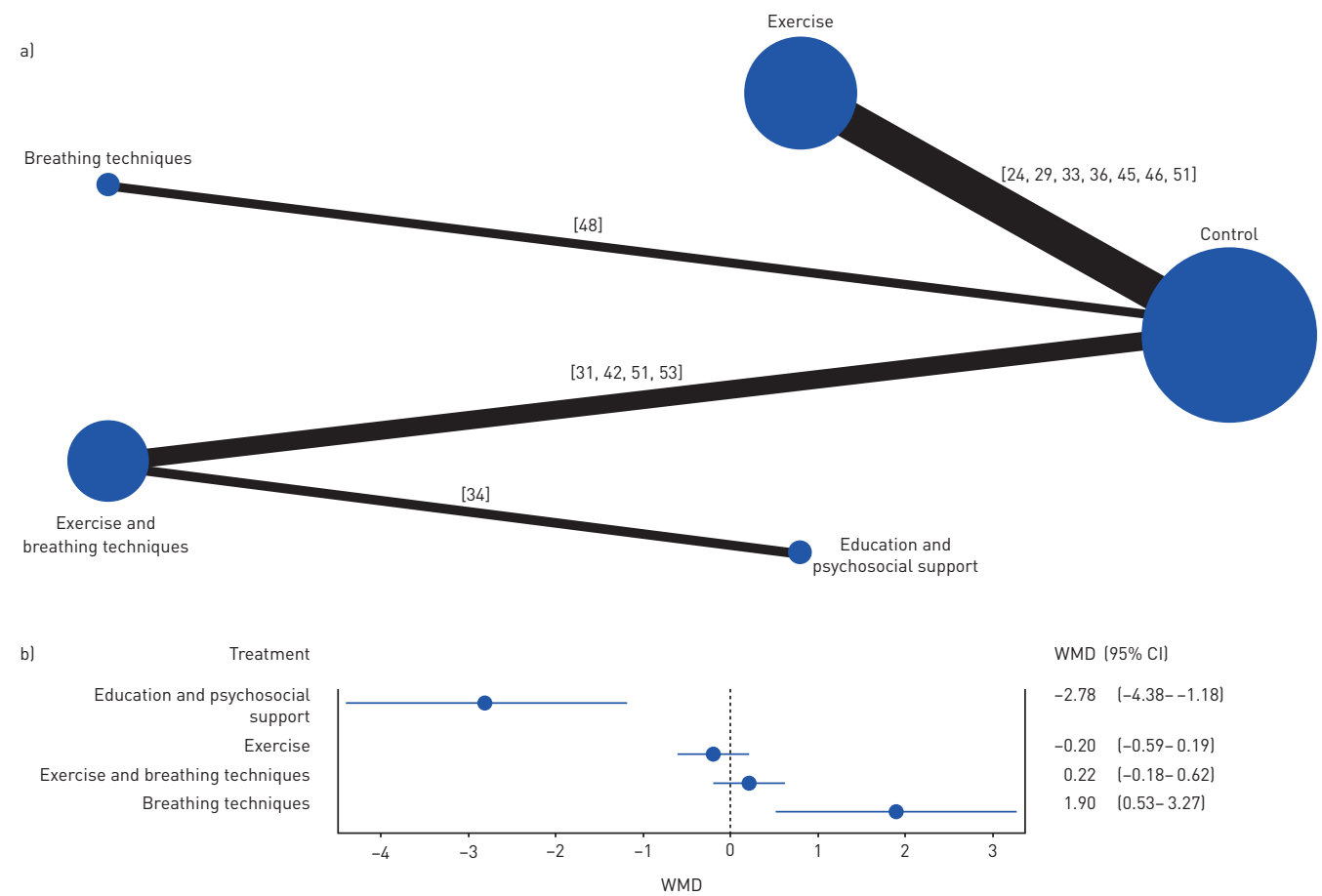

FIGURE 3 Effects of the different interventions on dyspnoea measured with the modified Borg scale. a) Network plot of treatments with nodes representing the number of interventions being compared and edges representing the available direct comparisons (comparisons evaluated in at least one study) between pairs of interventions. b) Forest plot of network meta-analysis results (all interventions versus standard of care). WMD: weighted mean difference.
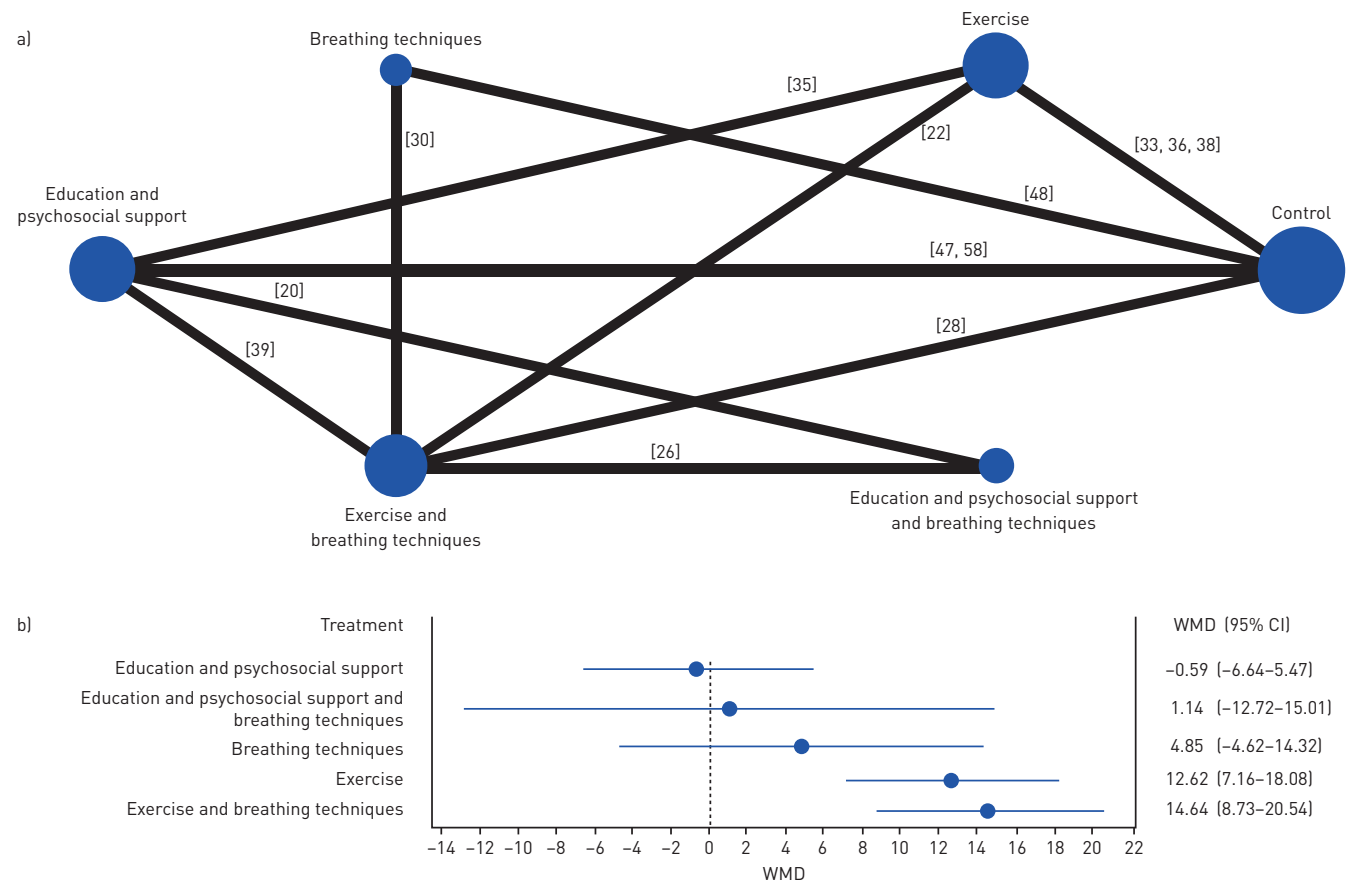

FIGURE 4 Effects of the different interventions on health-related quality of life measured with the St George's respiratory questionnaire. a) Network plot of treatments with nodes representing the number of interventions being compared and edges representing the available direct comparisons (comparisons evaluated in at least one study) between pairs of interventions. b) Forest plot of network meta-analysis results (all interventions versus standard of care). 


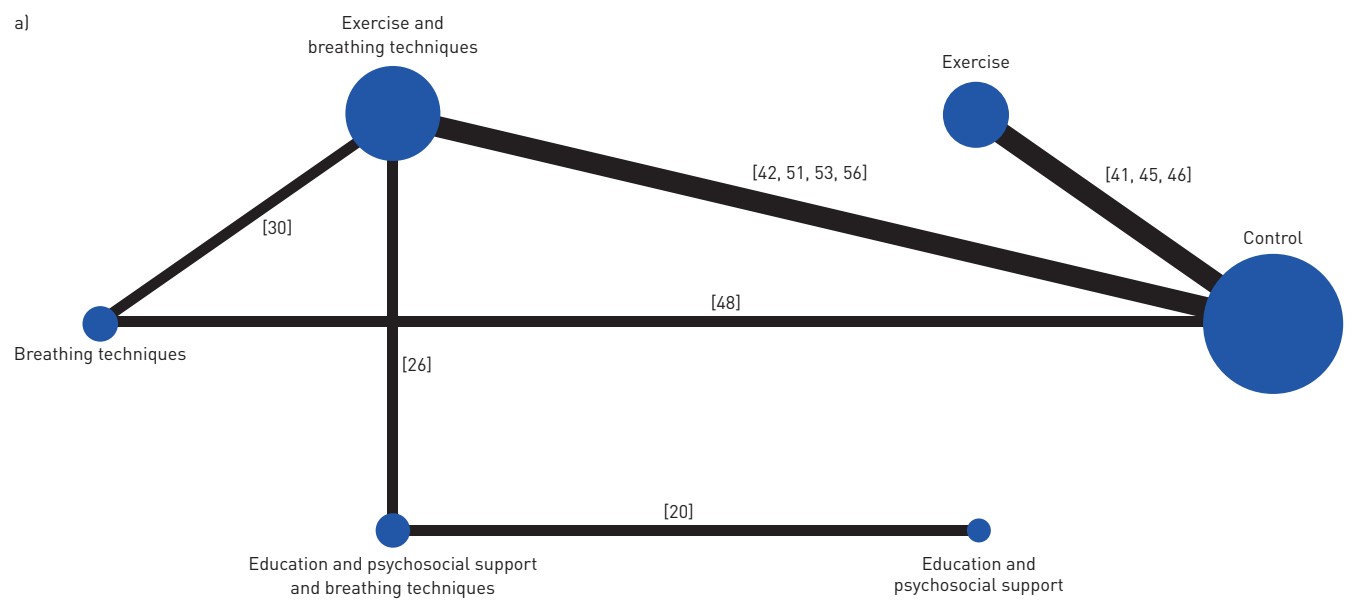
breathing techniques

Exercise

Exercise and breathing techniques Breathing techniques

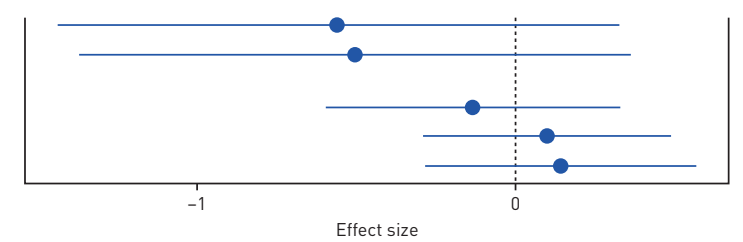

Effect size $(95 \% \mathrm{Cl})$

$-0.56(-1.44-0.33)$

$-0.50(-1.37-0.37)$

$-0.13(-0.59-0.33)$

$0.11(-0.28-0.50)$

$0.15(-0.28-0.57)$

FIGURE 5 Effects of the different interventions on length of hospitalisation measured in number of days. a) Network plot of treatments with nodes representing the number of interventions being compared and edges representing the available direct comparisons (comparisons evaluated in at least one study) between pairs of interventions. b) Forest plot of network meta-analysis results (all interventions versus standard of care).

Borg scale in patients with AECOPD receiving standard of care [65]. In terms of length of the hospitalisation, breathing techniques and the combination of exercise with breathing techniques resulted in an overall positive effect in reducing the number of days hospitalised. Furthermore, patient's knowledge was one of the outcome measures presenting larger ES, showing how empowering pulmonary rehabilitation can be during AECOPD. It has been recognised that many AECOPD occur without being reported to health professionals [1]. Despite the short duration of these events, they have a negative impact on patients' health status, thus highlighting the importance of educating these patients [1]. During an AECOPD, patients are more willing to change their behaviour and, therefore, there is a window of opportunity for patients' education that it is important not to miss but which should be optimised to prevent patients' clinical deterioration [28, 66, 67]. Based on all these findings, future studies should consider a combination of exercise training, breathing techniques and education and psychosocial support as core components of pulmonary rehabilitation in patients with AECOPD.

Different lengths of intervention programmes as well as different frequencies and durations of sessions were found. Short-term programmes presented superior results to standard of care and seem to improve exercise capacity more than programmes of longer duration. Nevertheless, these results must be interpreted with caution as groups of studies for short- and long-term programmes presented marked differences. Studies applying short-term programmes [22, 26, 31, 34] were all inpatient studies and thus started their programmes earlier in the rehabilitation phase (up to the third day of hospital admission), used daily or twice daily sessions in their interventions and were all supervised. Studies applying long-term programmes [28, 39] started their programmes later in the rehabilitation process, only at hospital discharge or 2 weeks after discharge, used a smaller frequency of sessions and in one of the studies most of the sessions were not even supervised. All these aspects, together with the small number of studies and the uncertainty about the period needed for a patient completely recover from an exacerbation $[1,62]$, might have contributed to the diversity between studies and influenced the dose [68] and effectiveness of the programmes implemented. Therefore, the most appropriate length of pulmonary rehabilitation as well as frequency and duration of its sessions during AECOPD are yet to be determined.

Most studies lacked information about the intensity and progressions used in their interventions. It is known that training intensity influences the physiological responses to the training and that the minimum level of intensity to reach benefits varies due to many factors, in particular a person's health status [68]. 
TABLE 2 Most frequently used and effective designs for delivering pulmonary rehabilitation during acute exacerbations of COPD

\begin{tabular}{|c|c|c|}
\hline Outcome & Most effective intervention & Pooled effects $(95 \% \mathrm{Cl})$ \\
\hline Exercise capacity: 6MWT & Exercise + breathing techniques & WMD $-41.06(-131.70-49.58)$ \\
\hline Dyspnoea symptoms: modified Borg scale & Breathing techniques & WMD $1.90(0.53-3.27)$ \\
\hline Health-related quality of life: SGRQ & Exercise + breathing techniques & WMD 14.64 (8.73-20.54) \\
\hline Length of hospitalisation: number of days & Breathing techniques & ES $0.15(-0.28-0.57)$ \\
\hline
\end{tabular}

Inpatient setting within $24-48 \mathrm{~h}$ of hospital admission. Components are exercise training, education and psychosocial support and breathing techniques. 6MWT: 6-min walk test; WMD: weighted mean difference: ES: effect size; SGRQ: St George's Respiratory Questionnaire.

Therefore, studies comparing different levels of training intensities during AECOPD are needed to establish recommendations about this aspect of the design.

Most of the available evidence published has described hospitalised patients, who are known to present more severe exacerbations and/or have more severe underlying disease than those managed on an outpatient basis [1]. Since $>80 \%$ of patients with AECOPD are managed at outpatient settings [1], future research is warranted on patients with AECOPD who are managed on an outpatient basis, i.e. in hospital outpatient departments, community and home.

Overall, the interventions applied had few adverse effects and the three more serious events found were related to the underlying comorbidities of the included patients [22, 40]. Thus, implementation of pulmonary rehabilitation programmes during AECOPD seems a safe approach.

\section{Methodological considerations}

This systematic review and meta-analysis has a number of strengths and limitations that need to be acknowledged. A thorough systematic search and screening was performed, and the most well-established guideline for the conductance of systematic reviews (PRISMA) was followed. Additionally, the performance of a network meta-analysis allowed the direct and indirect comparison of multiple aspects of the design, and has been recommended as a powerful procedure that provides more precise estimates [69]; however, the framework used for the analysis is not able to include all data from trials with more than two arms at once. Moreover, analysis of the designs of pulmonary rehabilitation programmes was found to be highly challenging due to the large diversity of interventions, outcome measures and units of measurement found and the lack of clarity in treatment description. All these aspects have hampered the results synthesis and more detailed comparisons. Finally, the definition of standard care was not always clearly described, which might have also influenced some of the pooled results.

\section{Conclusions}

Pulmonary rehabilitation is a safe intervention during AECOPD. The combination of exercise, breathing techniques and education and psychosocial support seems to be the most effective for improvements on dyspnoea, knowledge about the disease, exercise capacity, HRQoL and length of hospitalisation, and thus all these components might be considered as core components to implement pulmonary rehabilitation during AECOPD (table 2). Studies may now focus on exploring comparisons among different timings, lengths of intervention, durations and frequencies of sessions, and intensities of exercise training to allow the establisahment of clear recommendations regarding these aspects of the design.

Conflict of interest: None declared.

Support statement: This work was funded by Programa Operacional de Competitividade e Internacionalização (POCI) through Fundo Europeu de Desenvolvimento Regional (FEDER: POCI-01-0145-FEDER-007628) and Fundação para a Ciência e Tecnologia (PTDC/DTP-PIC/2284/2014, PTDC/SAL-SER/28806/2017, SFRH/BD/147200/2019, UIDB/04501/ 2020, UIDB/04106/2020 and UIDP/04106/2020).

\section{References}

1 The Global Initiative for Chronic Obstructive Lung Disease. Global Strategy for the Diagnosis, Management and Prevention of COPD. 2019.

2 Wedzicha JA, Miravitlles M, Hurst JR, et al. Management of COPD exacerbations: a European Respiratory Society/American Thoracic Society guideline. Eur Respir J 2017; 49: 1600791. 
3 Rochester CL, Vogiatzis I, Holland AE, et al. An official American Thoracic Society/European Respiratory Society policy statement: enhancing implementation, use, and delivery of pulmonary rehabilitation. Am J Respir Crit Care Med 2015; 192: 1373-1386.

4 Spruit MA, Singh SJ, Garvey C, et al. An official American Thoracic Society/European Respiratory Society statement: key concepts and advances in pulmonary rehabilitation. Am J Respir Crit Care Med 2013; 188: e13-e64.

5 Puhan MA, Gimeno-Santos E, Cates CJ, et al. Pulmonary rehabilitation following exacerbations of chronic obstructive pulmonary disease. Cochrane Library 2016; 12: CD005305.

6 Spruit MA, Singh SJ, Rochester CL, et al. Pulmonary rehabilitation for patients with COPD during and after an exacerbation-related hospitalisation: back to the future? Eur Respir J 2018; 51: 1701312.

7 Seemungal TA, Donaldson GC, Bhowmik A, et al. Time course and recovery of exacerbations in patients with chronic obstructive pulmonary disease. Am J Respir Crit Care Med 2000; 161: 1608-1613.

8 Cydulka RK, McFadden Jr E, Emerman CL, et al. Patterns of hospitalization in elderly patients with asthma and chronic obstructive pulmonary disease. Am J Respir Crit Care Med 1997; 156: 1807-1812.

9 Moher D, Liberati A, Tetzlaff J, et al. Preferred reporting items for systematic reviews and meta-analyses: the PRISMA statement. PLoS Med 2009; 6: e1000097.

10 Verhagen AP, de Vet HC, de Bie RA, et al. The Delphi list: a criteria list for quality assessment of randomized clinical trials for conducting systematic reviews developed by Delphi consensus. J Clin Epidemiol 1998; 51: $1235-1241$.

11 Verhagen AP, de Bie RA, Lenssen AF, et al. Impact of quality items on study outcome: treatments in acute lateral ankle sprains. Int J Technol Assess Health Care 2000; 16: 1136-1146.

12 Kingma JJ, de Knikker $\mathrm{R}$, Wittink $\mathrm{H}$, et al. Eccentric overload training in patients with chronic Achilles tendinopathy: a systematic review. Br J Sports Med 2007; 41: e3.

13 Landis JR, Koch GG. The measurement of observer agreement for categorical data. Biometrics 1977; 33: 159-174.

14 Morris SB. Estimating effect sizes from pretest-posttest-control group designs. Organ Res Methods 2008; 11: 364-386.

15 Cohen J. Statistical power analysis for the behavioral sciences. 2nd Edn. Hillsdale, Erlbaum Associates, 1988.

16 Doi SA, Barendregt JJ. A generalized pairwise modelling framework for network meta-analysis. Int J Evid Based Healthc 2018; 16: 187-194.

17 Smith LJW, Beretvas SN. Estimation of the standardized mean difference for repeated measures designs. J Mod Appl Stat Methods 2009; 8: 27.

18 Benzo R, Vickers K, Novotny PJ, et al. Health coaching and chronic obstructive pulmonary disease rehospitalization. A randomized study. Am J Respir Crit Care Med 2016; 194: 672-680.

19 Casas A, Troosters T, Garcia-Aymerich J, et al. Integrated care prevents hospitalisations for exacerbations in COPD patients. Eur Respir J 2006; 28: 123-130.

20 Cross JL, Elender F, Barton G, et al. Evaluation of the effectiveness of manual chest physiotherapy techniques on quality of life at six months post exacerbation of COPD (MATREX): a randomised controlled equivalence trial. BMC Pulm Med 2012; 12: 33.

21 Johnson-Warrington V, Rees $\mathrm{K}$, Gelder C, et al. Can a supported self-management program for COPD upon hospital discharge reduce readmissions? A randomized controlled trial. Int J Chron Obstruct Pulmon Dis 2016; 11: 1161-1169.

22 Osadnik CR, McDonald CF, Miller BR, et al. The effect of positive expiratory pressure (PEP) therapy on symptoms, quality of life and incidence of re-exacerbation in patients with acute exacerbations of chronic obstructive pulmonary disease: a multicentre, randomised controlled trial. Thorax 2014; 69: 137-143.

23 Puhan MA, Spaar A, Frey M, et al. Early versus late pulmonary rehabilitation in chronic obstructive pulmonary disease patients with acute exacerbations: a randomized trial. Respiration 2012; 83: 499-506.

24 Ali MS, Talwar D, Jain S. The effect of a short-term pulmonary rehabilitation on exercise capacity and quality of life in patients hospitalised with acute exacerbation of chronic obstructive pulmonary disease. Indian J Chest Dis Allied Sci 2014; 56: 13-19.

25 Babu AS, Noone MS, Haneef M, et al. The effects of "on-call/out of hours" physical therapy in acute exacerbations of chronic obstructive pulmonary disease: a randomized controlled trial. Clin Rehabil 2010; 24: 802-809.

26 Borges RC, Carvalho CR. Impact of resistance training in chronic obstructive pulmonary disease patients during periods of acute exacerbation. Arch Phys Med Rehabil 2014; 95: 1638-1645.

27 Chaplin EJL, Houchen L, Greening NJ, et al. Neuromuscular stimulation of quadriceps in patients hospitalised during an exacerbation of COPD: a comparison of low $(35 \mathrm{~Hz})$ and high $(50 \mathrm{~Hz})$ frequencies. Physiother Res Int 2013; 18: 148-156.

28 Deepak T, Mohapatra P, Janmeja A, et al. Outcome of pulmonary rehabilitation in patients after acute exacerbation of chronic obstructive pulmonary disease. Indian J Chest Dis Allied Sci 2014; 56: 7-12.

29 Eaton T, Young P, Fergusson W, et al. Does early pulmonary rehabilitation reduce acute health-care utilization in COPD patients admitted with an exacerbation? A randomized controlled study. Respirology 2009; 14: 230-238.

30 Greulich T, Nell C, Koepke J, et al. Benefits of whole body vibration training in patients hospitalised for COPD exacerbations-a randomized clinical trial. BMC Pulm Med 2014; 14: 60.

31 He M, Yu S, Wang L, et al. Efficiency and safety of pulmonary rehabilitation in acute exacerbation of chronic obstructive pulmonary disease. Med Sci Monit 2015; 21: 806-812.

32 Kirsten D, Taube C, Lehnigk B, et al. Exercise training improves recovery in patients with COPD after an acute exacerbation. Respir Med 1998; 92: 1191-1198.

33 Ko FW, Dai DL, Ngai J, et al. Effect of early pulmonary rehabilitation on health care utilization and health status in patients hospitalized with acute exacerbations of COPD. Respirology 2011; 16: 617-624.

34 Liao L-Y, Chen K-M, Chung W-S, et al. Efficacy of a respiratory rehabilitation exercise training package in hospitalized elderly patients with acute exacerbation of COPD: a randomized control trial. Int J Chron Obstruct Pulmon Dis 2015; 10: 1703-1709.

35 Man WD, Polkey MI, Donaldson N, et al. Community pulmonary rehabilitation after hospitalisation for acute exacerbations of chronic obstructive pulmonary disease: randomised controlled study. BMJ Open 2004; 329: 1209.

36 Murphy N, Bell C, Costello RW. Extending a home from hospital care programme for COPD exacerbations to include pulmonary rehabilitation. Respir Med 2005; 99: 1297-1302. 
37 Nava S. Rehabilitation of patients admitted to a respiratory intensive care unit. Arch Phys Med Rehabil 1998; 79: 849-854.

38 Seymour JM, Moore L, Jolley CJ, et al. Outpatient pulmonary rehabilitation following acute exacerbations of COPD. Thorax 2010; 65: 423-428.

39 Song HY, Yong SJ, Hur HK. Effectiveness of a brief self-care support intervention for pulmonary rehabilitation among the elderly patients with chronic obstructive pulmonary disease in Korea. Rehabil Nurs 2014; 39: 147-156.

40 Tang CY, Blackstock FC, Clarence M, et al. Early rehabilitation exercise program for inpatients during an acute exacerbation of chronic obstructive pulmonary disease: a randomized controlled trial. J Cardiopulm Rehabil Prev 2012; 32: 163-169.

41 Torres-Sánchez I, Valenza MC, Cabrera-Martos I, et al. Effects of an exercise intervention in frail older patients with chronic obstructive pulmonary disease hospitalized due to an exacerbation: a randomized controlled trial. COPD 2017; 14: 37-42.

42 Torres-Sánchez I, Valenza MC, Sáez-Roca G, et al. Results of a multimodal program during hospitalization in obese COPD exacerbated patients. COPD 2016; 13: 19-25.

43 Troosters T, Probst VS, Crul T, et al. Resistance training prevents deterioration in quadriceps muscle function during acute exacerbations of chronic obstructive pulmonary disease. Am J Respir Crit Care Med 2010; 181: 1072-1077.

44 Kjærgaard JL, Juhl CB, Lange P, et al. Early pulmonary rehabilitation after acute exacerbation of COPD: a randomised controlled trial. ERJ Open Res 2020; 6: 00173-2019.

45 Lopez-Lopez L, Torres-Sanchez I, Rodriguez-Torres J, et al. Randomized feasibility study of twice a day functional electrostimulation in patients with severe chronic obstructive pulmonary disease hospitalized for acute exacerbation. Physiother Theory Pract 2019: in press [https://doi.org/10.1080/09593985.2019.1694611].

46 Lopez-Lopez L, Valenza MC, Rodriguez-Torres J, et al. Results on health-related quality of life and functionality of a patient-centered self-management program in hospitalized COPD: a randomized control trial. Disabil Rehabil 2019: in press [https://doi.org/10.1080/09638288.2019.1609099].

47 Garcia-Aymerich J, Hernandez C, Alonso A, et al. Effects of an integrated care intervention on risk factors of COPD readmission. Respir Med 2007; 101: 1462-1469.

48 Kodric M, Garuti G, Colomban M, et al. The effectiveness of a bronchial drainage technique (ELTGOL) in COPD exacerbations. Respirology 2009; 14: 424-428.

49 Eastwood B, Jepsen N, Coulter K, et al. Challenges of undertaking a clinical trial using bubble-PEP in an acute exacerbation of chronic obstructive pulmonary disease: a feasibility study. NZ J Physiother 2016; 44: 8-16.

50 Basri R, Tahir M, Naseem M. Short-term effects of chest physiotherapy in acute exacerbation of chronic obstructive pulmonary disease. J Med Sci 2017; 25: 323-327.

51 Torres-Sánchez I, Valenza MC, Cebriá i Iranzo M, et al. Effects of different physical therapy programs on perceived health status in acute exacerbation of chronic obstructive pulmonary disease patients: a randomized clinical trial. Disabil Rehabil 2018; 40: 2025-2031.

52 Yohannes AM, Connolly MJ. Early mobilization with walking aids following hospital admission with acute exacerbation of chronic obstructive pulmonary disease. Clin Rehabil 2003; 17: 465-471.

53 Lopez LL, Santiago MG, Galindo MD, et al. Efficacy of combined electrostimulation in patients with acute exacerbation of COPD: randomised clinical trial. Med Clin 2018; 151: 323-328.

54 Bellone A, Spagnolatti L, Massobrio M, et al. Short-term effects of expiration under positive pressure in patients with acute exacerbation of chronic obstructive pulmonary disease and mild acidosis requiring non-invasive positive pressure ventilation. Intensive Care Med 2002; 28: 581-585.

55 Valenza MC, Valenza-Peña G, Torres-Sánchez I, et al. Effectiveness of controlled breathing techniques on anxiety and depression in hospitalized patients with COPD: a randomized clinical trial. Respir Care 2014; 59: 209-215.

56 Hernandez C, Casas A, Escarrabill J, et al. Home hospitalisation of exacerbated chronic obstructive pulmonary disease patients. Eur Respir J 2003; 21: 58-67.

57 Lainscak M, Kadivec S, Kosnik M, et al. Discharge coordinator intervention prevents hospitalizations in patients with COPD: a randomized controlled trial. J Am Med Dir Assoc 2013; 14: 450.

58 Aboumatar H, Naqibuddin M, Chung S, et al. Effect of a hospital-initiated program combining transitional care and long-term self-management support on outcomes of patients hospitalized with chronic obstructive pulmonary disease: a randomized clinical trial. JAMA 2019; 322: 1371-1380.

59 Janaudis-Ferreira T, Carr SJ, Harrison SL, et al. Can patients with COPD assimilate disease-specific information during an acute exacerbation? Results of a pilot randomized controlled trial. Chest 2018; 154: 588-596.

60 McCarthy B, Casey D, Devane D, et al. Pulmonary rehabilitation for chronic obstructive pulmonary disease. Cochrane Library 2015; 2: CD003793.

61 Anzueto A. Impact of exacerbations on COPD. Eur Respir Rev 2010; 19: 113-118.

62 Wedzicha JA, Seemungal TA. COPD exacerbations: defining their cause and prevention. Lancet 2007; 370: 786-796.

63 Holland AE, Spruit MA, Troosters T, et al. An official European Respiratory Society/American Thoracic Society technical standard: field walking tests in chronic respiratory disease. Eur Respir J 2014; 44: 1428-1446.

64 Schünemann HJ, Griffith L, Jaeschke R, et al. Evaluation of the minimal important difference for the feeling thermometer and the St. George's Respiratory Questionnaire in patients with chronic airflow obstruction. J Clin Epidemiol 2003; 56: 1170-1176.

65 Oliveira A, Machado A, Marques A. Minimal important and detectable differences of respiratory measures in outpatients with AECOPD. COPD 2018; 15: 479-488.

66 Puhan MA, Gimeno-Santos E, Scharplatz M, et al. Pulmonary rehabilitation following exacerbations of chronic obstructive pulmonary disease. Cochrane Database Syst Rev 2011; 10: CD005305.

67 Oliveira A, Marques A. Exploratory mixed methods study of respiratory physiotherapy for patients with lower respiratory tract infections. Physiotherapy 2016; 102: 111-118.

68 American College of Sports Medicine. ACSM's guidelines for exercise testing and prescription. Philadelphia, Lippincott Williams \& Wilkins, 2013.

69 Bagg MK, Salanti G, McAuley JH. Comparing interventions with network meta-analysis. J Physiother 2018; 64 $128-132$. 